\title{
Síndrome do comer noturno: aspectos conceituais, epidemiológicos, diagnósticos e terapêuticos
}

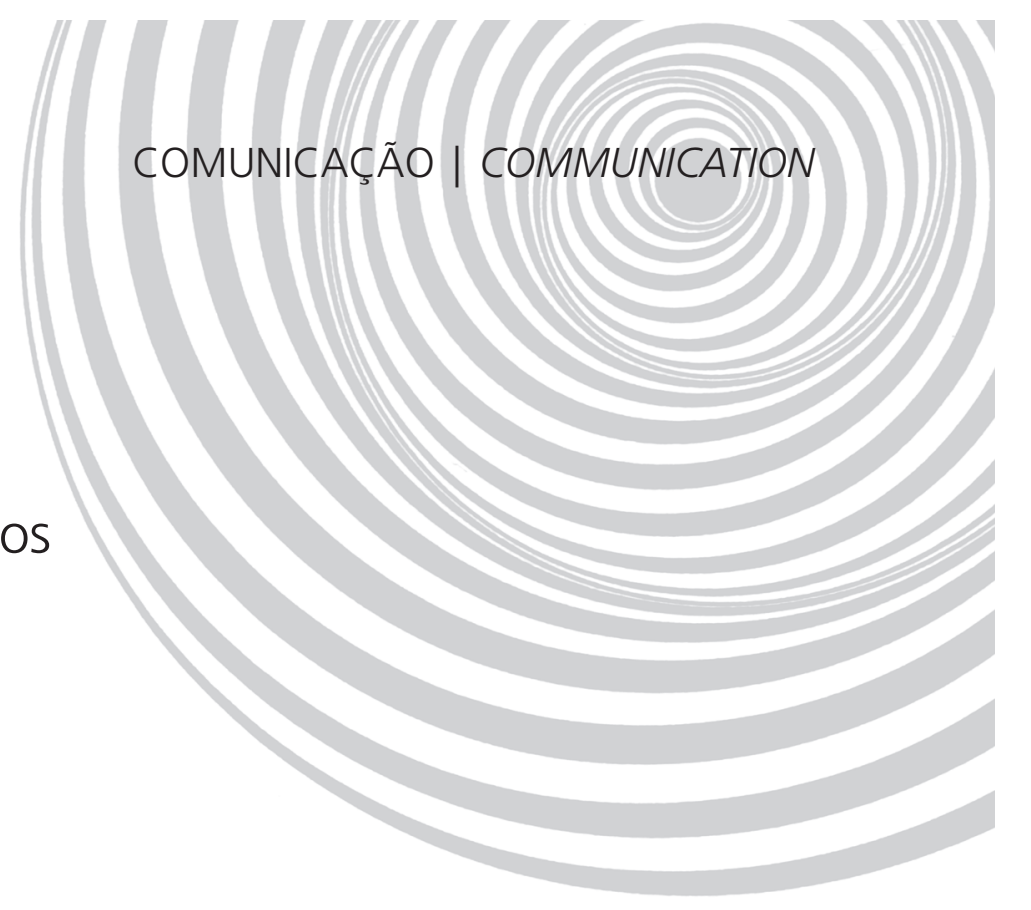

\author{
Night eating syndrome: conceptual, \\ epidemiological, diagnostic and \\ therapeutic aspects
}

\author{
Ana Beatriz Cauduro HARB ${ }^{1,2}$ \\ Wolnei $\mathrm{CAUMO}^{3}$ \\ Priscila RAUPP \\ Maria Paz Loayza HIDALGO ${ }^{5}$
}

\section{RE S U M O}

O comportamento e o padrão alimentar são complexos, envolvendo aspectos metabólicos, fisiológicos e ambientais, e apresentando ritmicidade circadiana, herdada e espécie específica, sendo a humana essencialmente diurna. Este estudo tem como objetivo descrever a Síndrome do Comer Noturno, caracterizada por um atraso circadiano do padrão alimentar, mediado por alterações neuroendócrinas ao estresse. Procedeu-se à revisão da bibliografia existente, a partir do exame de artigos publicados pela literatura internacional nas bases de dados dos sites Pubmed, Lilacs, Sirus, referentes ao período de 1955 até as últimas publicações em 2007. Realizou-se um levantamento bibliográfico usando os seguintes descritores: night eating syndrome, sleep, circadian rhythm, appetite, nocturnal eating. Foram localizados 74 artigos e destes foram selecionados 26, cujo tema central era a Síndrome do Comer Noturno. Apesar dos estudos realizados, ainda existe longo percurso a ser percorrido para compreender a gênese da Síndrome do Comer Noturno e as relações intrínsecas desta com outros processos fisiopatogênicos. Tendo em conta que esta síndrome está vinculada ao controle da fome e da saciedade e à dessincronização entre o ritmo alimentar e o ritmo sono/vigília, a compreensão do seu processo gênico poderá demonstrar o impacto da dessincronização dos ritmos circadianos da alimentação no processo saúde-doença, e auxiliar a compreensão de fatores implicados no índice crescente de obesidade da sociedade moderna.

Termos de indexação: Apetite. Comer à noite. Síndrome do Comer Noturno. Sono. Ritmo circadiano.

$\overline{1}$ Universidade do Vale do Rio dos Sinos, Curso de Nutrição. São Leopoldo, RS, Brasil.

2 Universidade Federal do Rio Grande do Sul, Hospital das Clínicas de Porto Alegre, Departamento da Psiquiatria. R. Ramiro Barcelos, 2350, Bom Fim, 90035-903, Porto Alegre, RS, Brasil. Correspondência para/Correspondence to: A.B.C. HARB. E-mail: <anaharb@anaharb.com.br>.

${ }^{3}$ Universidade Federal do Rio Grande do Sul, Instituto de Ciências Básicas da Saúde, Departamento de Farmacologia. Porto Alegre, RS, Brasil.

${ }^{4}$ Acadêmica, Universidade Federal do Rio Grande do Sul, Curso de Medicina. Porto Alegre, RS, Brasil.

5 Universidade Federal do Rio Grande do Sul, Faculdade de Medicina, Departamento de Psiquiatria e Medicina Legal. Porto Alegre, RS, Brasil. 


\title{
A B S T R A C T
}

\begin{abstract}
Behavior and eating patterns are complex, involve metabolic, physiological and environmental aspects and have inherited and species-specific circadian rhythmicity, with humans being essentially diurnal. The aim of this study is to describe the night eating syndrome, characterized by a delay of the circadian eating patterns and mediated by neuroendocrine stress. A review of the literature found in the Pubmed, Lilacs and Sirus databases published between 1955 and 2007 was done. The keywords used were: night eating syndrome, sleep, circadian rhythm, appetite, nocturnal eating. A total of 74 articles were found of which 26 were selected, whose central theme was the night eating syndrome. Despite all the studies done, there is still long way to go to understand the genesis of the night eating syndrome and its intrinsic relation with other physiological and pathogenic processes. Given that this syndrome is linked to the control of hunger and satiety and to the desynchronization between the eating rhythm and sleep / wake rhythm, understanding its genetic process can reveal the impact that the desynchronization of circadian eating rhythms has on the health-disease process and help understand the factors involved in the rising rates of obesity in modern society.
\end{abstract}

Indexing terms: Appetite. Nocturnal eating. Night Eating Syndrome. Sleep. Circadian rhythm.

\section{N T R O D U Ç Ã O}

O comportamento alimentar é complexo, envolvendo aspectos metabólicos, fisiológicos e ambientais ${ }^{1,2}$, que apresentam ritmicidade circadiana, herdada e espécie específica, sendo a humana essencialmente diurna. Está sincronizado ao ciclo claro/escuro e aos níveis de cortisol, serotonina, leptina, citocinas, entre outros ${ }^{2-5}$. Além disso, o ritmo social imprime marcado efeito na regulação da alimentação, pois a sociedade contemporânea funciona 24 horas por dia e seu impacto na quantidade, qualidade e nos horários da alimentação tem sido inexorável. Como exemplo, cita-se o atraso da alimentação para adaptação aos horários de trabalho³. A observação do comportamento alimentar induziu a definição de uma síndrome denominada Síndrome do Comer Noturno (SCN), caracterizada por um atraso circadiano do padrão alimentar ${ }^{6}$, mediado por alterações neuroendócrinas ${ }^{6-8}$. Dentre os critérios diagnósticos dessa síndrome relacionam-se: anorexia matutina; ingestão $\geq 50 \%$ da energia diária após as 19h; despertar para comer ao menos uma vez por noite nos últimos três meses, com consciência do ato; consumo de lanches de alto valor energético nos despertares noturnos; ausência de critérios para bulimia nervosa ou binge eating disorder ${ }^{7}$.

O histórico sobre o início das investigações que abordaram padrões e comportamentos alimentares remonta à década de 1950, quando ocorreu a primeira publicação que definia transtornos alimentares 9 . Isso motivou a busca dos possíveis mecanismos implicados no controle da fome e da saciedade. Essas observações apontavam para um processo passivo. Estudos subsequentes mostraram mecanismos fisiológicos que induzem e que controlam a fome e a saciedade de maneira ativa ${ }^{10}$. Além dos mecanismos reguladores fisiológicos inatos, as adaptações às exigências do meio imprimiram marcas no modo de viver. Isso é evidente pela a evolução do comportamento alimentar do homem ao longo do tempo. As mudanças têm exigido grande capacidade de adaptação física, mental e social. Por conseguinte, tornaram-se mais frequentes no cotidiano da vida moderna maiores níveis de estresse, ansiedade, alteração do ritmo do sono, tensão e alterações do padrão alimentar.

A Síndrome do Comer Noturno, conceituada como um atraso no ritmo circadiano do padrão alimentar ${ }^{6}$, certamente decorre dessa mudança social e comportamental. Tendo em conta que pesquisas recentes têm demonstrado uma possível relação intrínseca entre essa síndrome e o processo saúde-doença, como maior incidência de obesidade e diabetes, sobrepondo-se a uma pobre qualidade de vida, acredita-se que ainda existe espaço para um estudo de revisão, com o 
intuito de analisar as evidências conceituais, funcionais, de efeito e de intervenção até então alcançadas sobre a Síndrome do Comer Noturno.

\section{M É T O D O S}

Para alcançar o objetivo proposto, realizou-se uma busca sistematizada das informações nas bases de dados Pubmed, Lilacs, Sirus, referente ao período de 1955 (publicação do primeiro artigo sobre o tema) até o ano de 2007. A busca envolveu a revisão de capítulos de livros da área especializada, consulta à lista de referências dos mesmos e dos artigos selecionados para fundamentar o escopo dessa revisão.

Para apresentar os aspectos históricos, epidemiológicos, neuroendócrinos e diagnósticos foram usados como descritores: night eating syndrome, sleep, circadian rhythm, appetite, nocturnal eating. Foram localizados 74 artigos nas bases de dados consultadas. Destes, foram selecionados 26 artigos, que mencionavam no Abstract a Síndrome do Comer Noturno. Foram excluídos artigos escritos em outros idiomas que não o Inglês, Espanhol, Português, Francês e Italiano.

\section{Histórico}

Um dos pioneiros no estudo da SCN e dos transtornos alimentares foi Stunkard, com a primeira publicação em 1955, quando descreveu um padrão alimentar característico de certos pacientes obesos em uma clínica especializada no New York Hospital ${ }^{9}$. No curso do estudo sobre os transtornos alimentares, foram descritos, em 1959, a SCN e o binge eating syndrome, posteriormente denominado Binge Eating Disorder. Em 1979, o Binge Eating Disorder foi relacionado a vômitos e uso abusivo de laxativos, constituindo a bulimia nervosa descrita por Gerald Russell ${ }^{11}$.

Tanto a Síndrome do Comer Noturno como o Binge Eating Disorder são transtornos alimentares parecem fortemente associados ao sobrepeso e à obesidade. Diferem entre si pelo horário da ingestão de alimentos. O critério essencial do Binge Eating Disorder inclui episódios de superalimentação, acompanhados por subjetiva falta de controle, sem o uso de comportamentos compensatórios inapropriados. Na Síndrome do Comer Noturno, o critério essencial é o atraso de fase do padrão alimentar ${ }^{12,13}$.

Em 1996, Stunkard et al. ${ }^{11}$ revisaram a definição da SCN e especificaram que, para caracterizá-la, mais do que $50 \%$ da ingestão energética diária deveria ocorrer após as 19 horas. Sobretudo, deveriam existir anorexia matinal e problemas para iniciar ou para manter o sono. Essa síndrome foi descrita como uma resposta ao estresse circadiano, primeiramente observado em obesos. Também, foi observado que teria relação com o estresse e que os seus sintomas pareciam diminuir com o controle do mesmo ${ }^{6}$. No entanto, somente em 1999, Stunkard, juntamente com Birketvedt et al. ${ }^{7}$ iniciaram estudos sistemáticos da SCN usando o método comparativo, mostrando a diferença entre grupos de sujeitos que alocavam a maior parte da ingestão energética no turno da noite, com indivíduos-destituídos desse hábito e definidos como controles. A partir de então, foram demonstrados comportamentos e características neuroendócrinas específicas que permitiram diferenciar sujeitos classificados como portadores da Síndrome do Comer Noturno, daqueles controles.

Quanto à alocação dos alimentos, estudos observacionais mostraram que os sujeitos com a SCN consomem maior densidade energética diária do que os controles, com a alocação de $56 \%$ ou mais da ingestão energética entre 22 e 6 horas ${ }^{7}$. Demonstrou-se, também, que os sujeitos com SCN acordam significativamente mais vezes à noite do que os do grupo-controle, sendo que a metade dos despertares era acompanhada da ingestão de alimentos. Além disso, apresentavam maior nível de sintomas depressivos do que o grupo-controle ${ }^{14}$. No entanto, a Síndrome do Comer Noturno ainda não foi incorporada à lista de transtornos psiquiátricos de manuais diagnósticos, como o Diagnostic and Statistical Manual of Mental 
Disorders (DSM-IV R) e a Classificação Internacional de Doenças, $10^{a}$ revisão (CID-10).

\section{Aspectos epidemiológicos}

Estimativas atuais indicam que 0,5 a 1,5\% da população americana, incluindo adultos e crianças ${ }^{15,16}$ apresentam a SCN. Em clínicas de obesidade, os índices variam de 6 a 14\%; e se situam em $12 \%$, em sujeitos atendidos em clínicas de nutrição. A prevalência chega a $42 \%{ }^{17} \mathrm{em}$ pacientes que procuram cirurgia para obesidade nos EUA $^{18}$. No entanto, deve-se enfatizar que parte da variação na prevalência apresentada deve-se à diversidade de métodos para avaliar e definir a SCN. Os achados que parecem ser consensuais na definição da SCN são o aumento da adiposidade $^{19}$, observado em $57,1 \%$ dos obesos e em $28,6 \%$ dos indivíduos com sobrepeso ${ }^{19}$. Também tem sido reportada em $15 \%$ dos sujeitos que procuram tratamento para transtorno do comer compulsivo ${ }^{20}$. A associação entre a SCN e a obesidade ou o sobrepeso parece ganhar força ao observar que sua prevalência aumenta paralelamente ao ganho de peso, embora descrita também em sujeitos não obesos ${ }^{15}$. Apesar dos dados disponíveis não permitirem determinar uma relação do tipo causa-efeito, mostram, pelo menos, evidências consistentes sobre a associação dessa síndrome à obesidade ${ }^{20}$. Além disso, incorporam-se como características prevalentes da SCN o fato de ocorrer mais em mulheres, de aparecer na infância ou na adolescência ${ }^{20,16}$ e de ter conexão com comorbidades psiquiátricas ${ }^{21}$, especialmente depressão $(12,3 \%)$, uso de antipsicóticos atípicos e obesidade $(38,8 \%)^{21}$.

\section{Aspectos neuroendócrinos}

Os fatores neuroendócrinos da Síndrome do Comer Noturno se relacionam com alterações do ritmo circadiano de secreções endócrinas, como a do cortisol e da melatonina. Sendo a melatonina um neuro-hormônio regulatório do ritmo sono/vigília, pode-se postular, a partir dos resul- tados dos estudos de Birketvedt et al. ${ }^{7}$ e de O'Reardon et al. ${ }^{20}$ que parte dos sintomas apresentados por sujeitos com a SCN deve-se a baixos níveis de melatonina, pois incluem: dificuldades para adormecer ou se manter dormindo, assim como a má qualidade do sono. Tendo-se em conta que os níveis plasmáticos de melatonina são determinantes da regulação de um ritmo forte como o do sono/vigília, é razoável supor que a redução de melatonina possa contribuir para manter a insônia e o humor deprimido ${ }^{7}$. Adiciona-se a isso, o fato de os níveis plasmáticos noturnos da leptina serem mais baixos, o que pode contribuir para a menor inibição de impulsos de fome que levem à interrupção do sono. Também, dados de estudos comparativos mostraram que os níveis de cortisol diurnos se encontram mais elevados em sujeitos com SCN ${ }^{8}$.

\section{Critérios diagnósticos da SCN}

Os critérios para diagnóstico da síndrome ainda não estão claramente definidos. Existem sintomas importantes para caracterizá-la, embora provisórios. Dentre estes, relacionam-se: anorexia matutina; hiperfagia com ingestão de mais de 50\% do valor energético diário após as 19h; despertar, no mínimo, uma vez por noite nos últimos 3 três meses para comer lanches de alto valor energético. No entanto, o sujeito deve ter consciência do ato do comer noturno, mas não pode apresentar critérios para bulimia nervosa ou binge eating disorder ${ }^{7}$. Deve-se enfatizar que a duração e a frequência dos sintomas são mencionados em poucos estudos que tratam dos critérios diagnósticos, possivelmente, por existir divergências no critério de horário e do percentual da ingestão energética. Quanto à primeira, alguns autores mencionam como horário de referência após as 18,19 ou 20h, enquanto outros usam como referência após a refeição noturna ${ }^{20}$. Quanto à segunda, alguns autores definem que a alocação do percentual de ingestão energética para o período noturno deveria ser de, no mínimo, $25 \%$; outros de $50 \%$. 
Existem autores que omitem o percentual diário de energia ingerida, usando como prerrogativa a frequente sonegação de informação da ingestão diária de alimentos, especialmente por sujeitos obesos ${ }^{22}$. Apesar dessas divergências nos critérios para definir a SCN, na análise de itens preditivos que poderiam compor os critérios para o diagnóstico, dois apresentaram maior poder: ingestão de $25 \%$ ou mais do total diário de energia após a refeição noturna e a ingestão de alimentos nos despertares noturnos ${ }^{23}$. Adicionam-se outros critérios, cujo poder preditivo não é conhecido, como tensão à noite e/ou humor depressivo a partir da metade do dia ${ }^{20}$. Embora o estresse e os sintomas depressivos tenham sido observados e descritos consistentemente na clínica', ainda não foram incorporadas aos critérios diagnósticos da SCN. Os critérios comumente utilizados para o diagnóstico da SCN estão apresentados no Quadro 1.

Embora não haja consenso sobre o conjunto de sintomas que configuram o diagnóstico sindrômico da SCN, o que é consensual nas definições é o atraso no padrão circadiano da alocação da alimentação ${ }^{20}$. O apetite se caracteriza por estar diminuído na primeira metade do dia e aumentado no transcorrer do dia, associado à interrupção frequente do sono para comer ${ }^{20,22}$. Esses sintomas, possivelmente, expressam a dessincronização entre o ritmo alimentar e o ritmo sono/vigília'.

Apesar da ampla discussão sobre a síndrome, os critérios de diagnóstico não são universais, estando ainda em processo de construção. Vale ressaltar que existem instrumentos para diagnos- ticar a SCN utilizados no meio científico internacional e em processo de validação no Brasil. Por conseguinte, possivelmente no futuro poder-se-á aferir a SCN, tendo-se, inclusive, a possibilidade de estudar a alocação da maior parte da cota alimentar no curso do dia entre diferentes culturas e níveis sociais. É evidente que faz parte do processo diagnóstico diferenciar a SCN de outros transtornos alimentares, pois nem todos os sujeitos com queixa de comer à noite apresentam SCN. Pois a alocação da maior parte da fração alimentar para o turno da noite ocorre também em transtornos alimentares relacionados ao sono, como a sleep-related eating disorder, a bulimia nervosa e o transtorno do comer compulsivo'. Os critérios diagnósticos para diferenciar essas síndromes estão apresentados no Quadro 2.

A sleep-related eating disorder é uma parasonia, que caracteriza uma forma atípica de sonambulismo com pouca ou total falta de consciência associada aos episódios do comer noturno. Ocorre ao longo dos transtornos do sono, como nos transtornos periódicos dos movimentos das pernas, na síndrome das pernas inquietas, nos transtornos periódicos dos movimentos dos braços e na apnéia obstrutiva do sono. Os pacientes com sleep-related eating disorder costumam fazer combinações alimentares não habituais, como a escolha de alimentos bizarros ${ }^{20,24}$. A SCN diferencia-se também da bulimia nervosa e do transtorno do comer compulsivo porque os alimentos consumidos não são em grandes quantidades e não há comportamento típico de compensação. Tanto na

Quadro 1. Diferenças entre os critérios diagnósticos da Síndrome do Comer Noturno apresentados em 1955 e aqueles de $1999^{22}$.

\begin{tabular}{|c|c|c|}
\hline & Stunkard et al. ${ }^{9}$ & Birketvedt et al. ${ }^{7}$ \\
\hline Hiperfagia noturna & $\begin{array}{l}\text { Consumo de pelo menos } 25 \% \text { do total da ener- } \\
\text { gia diárias após a refeição noturna }\end{array}$ & $\begin{array}{l}\geq 50 \% \text { do total de energia diárias depois da refeição } \\
\text { noturna }\end{array}$ \\
\hline Anorexia matinal & $\begin{array}{l}\text { Anorexia matinal com negligência ao consumo de } \\
\text { alimentos no desjejum }\end{array}$ & Anorexia matinal, mesmo depois de ter feito o desjejum \\
\hline Inicio do sono & $\begin{array}{l}\text { Insone pelo menos até a meia noite, mais da meta- } \\
\text { de do tempo }\end{array}$ & \\
\hline Despertares & - & Pelo menos 1 vez por noite \\
\hline Comer noturno & - & Consumo de lanches durante os despertares \\
\hline
\end{tabular}


Quadro 2. Critérios para diferenciar a Síndrome do Comer Noturno ( $\mathrm{SCN}$ ) de Bulimia Nervosa (BN), Binge Eating Disorder (BED) e Sleep-Related Eating Disorder (SRED) ${ }^{22}$.

\begin{tabular}{llll}
\hline Sintomas & BN/BED & SCN & SRED \\
\hline Anorexia matinal & Usualmente não & Sim & Sim \\
Hiperfagia noturna & Não & Sim & Não \\
Hora de ocorrência das compulsões & Sim & Sim (à noite somente) & Não \\
Compulsões & Sim & Não (pequenos lanches) & Não (itens alimentares bizarros) \\
Ingestões noturnas & Raramente & Sim & Sim \\
Despertares noturnos para comer & Sim & Sim & Sem consciência dos despertares \\
Amnésia para os eventos & Não & Não & Sim \\
Estudos com polissonografia & Normal & Baixa eficiência do sono & Transtornos do sono: apnéia, per- \\
& & & nas inquietas, sonambulismo \\
Tratamento & Terapia cognitivo- & SSRls Topiramato & Agonista da dopamina, Topiramato \\
& -comportamental & & \\
\hline
\end{tabular}

SNC quanto no sleep-related eating disorder existe uma perda de controle (mais no primeiro do que na SCN), entretanto a diferença é quanto ao horário em que isto acontece (na SCN ocorre à noite, interrompendo o sono normal) $)^{8,20,22}$ enquanto que no sleep-related eating disorder pode ocorrer a qualquer hora do dia. Na SCN pode existir baixa autoestima, estresse excessivo $8,20,21$, sintomas ansiosos e humor depressivo, quando comparados aos sujeitos do grupo controle.

Em 1990, a Síndrome Noturna de Comer e Beber (NEDS) foi incluída na International Classification of Sleep Disorders of American Academy of Sleep Medicine como sendo uma dissonia extrínseca. Sua definição é caracterizada por recorrentes despertares com a inabilidade para retomar o sono sem comer ou beber algo. Isto ocorre com o indivíduo consciente, de forma não vinculada à existência de transtorno mental ou clínico ${ }^{22}$. Constituem-se critérios diagnósticos da NEDS os seguintes aspectos: A) O paciente se queixa de dificuldade de manter o sono; B) O paciente, frequentemente e recorrentemente, acorda para comer ou beber; C) Para iniciar o sono sente necessidade de ingerir a comida ou a bebida desejada; D) A polissonografia demonstra aumento no número ou na duração de despertares; E) Ausência de outra doença mental ou clinica e sem outra doença do sono.

\section{Tratamento}

O tratamento da SCN pode envolver intervenções farmacológicas e comportamentais. $\mathrm{Na}$ seleção da técnica, devem-se cotejar os parâmetros de eficácia e efetividade. Este segundo parâmetro inclui facilidade posológica, potenciais de interações e de efeitos adversos, experiência de uso e custo. O nível de evidência farmacológicoclínico, que classifica de forma hierarquizada a evidência científica nos enfoques de terapia, prevenção e etiologia/risco está apresentado no Quadro 3. Embora os sistemas em uso para classificação das evidências variem enormemente entre si, o sistema apresentado se baseia no proposto pelo Centre for Evidence Based Medicine de Oxford. Sua classificação difere de vários outros sistemas em voga no Brasil, por exigir definição mais precisa de certos aspectos dos ensaios clínicos randomizados (a definição dos desfechos como clínicos ou meramente como substitutos, por exemplo).

\section{Tratamentos fitoterápicos}

No tratamento da SCN é comum o uso de fitoterápicos, como exemplo a Kavain. Entra nesse espectro a melatonina, especialmente porque, em muitos países, incluindo os EUA, está classificada como suplemento alimentar e não como fármaco. 
Quadro 3. Níveis de evidência científica nos enfoques de terapia, prevenção e da etiologia/risco da Síndrome do Comer Noturno.

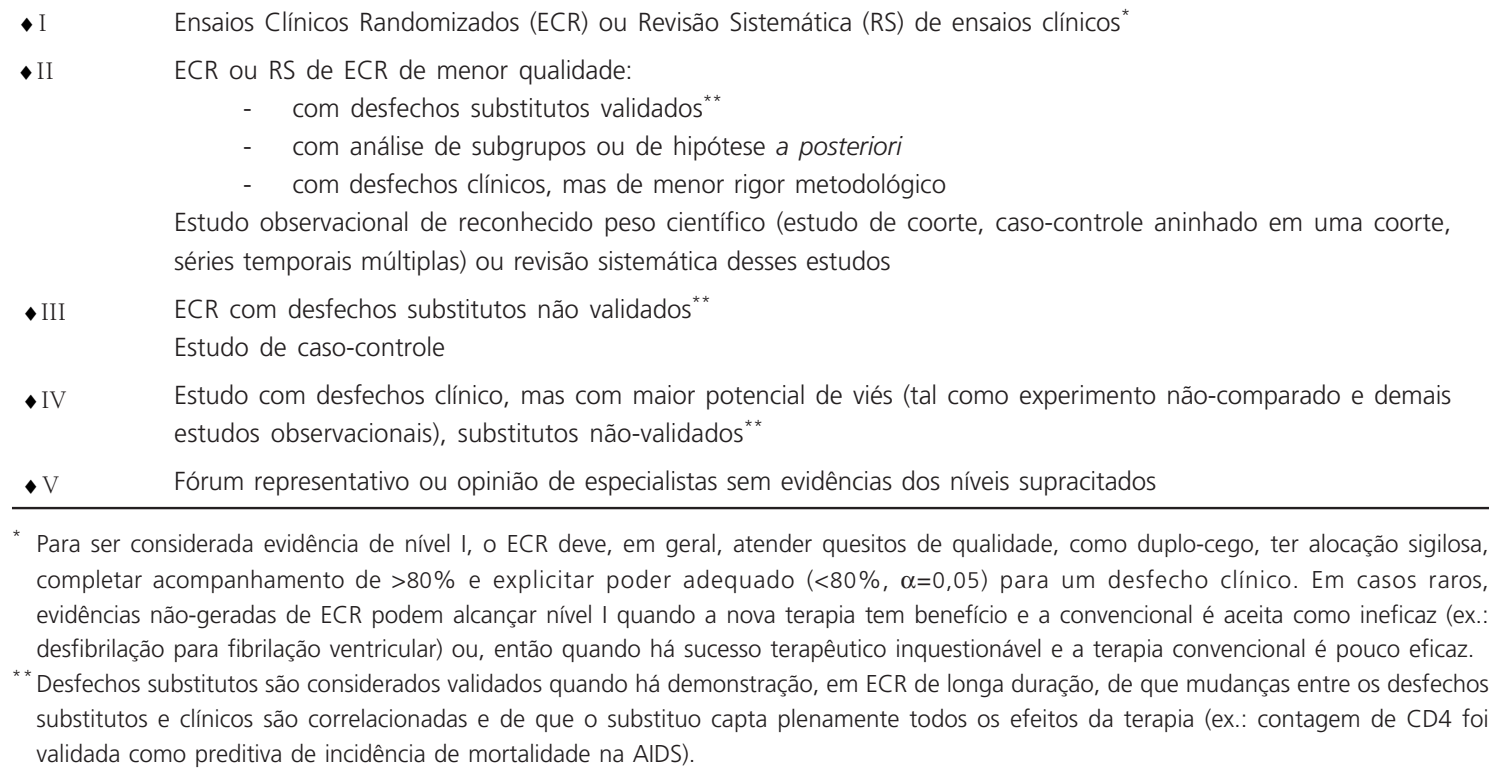

* Para ser considerada evidência de nível I, o ECR deve, em geral, atender quesitos de qualidade, como duplo-cego, ter alocação sigilosa, completar acompanhamento de $>80 \%$ e explicitar poder adequado $(<80 \%, \alpha=0,05)$ para um desfecho clínico. Em casos raros, evidências não-geradas de ECR podem alcançar nível I quando a nova terapia tem benefício e a convencional é aceita como ineficaz (ex.: desfibrilação para fibrilação ventricular) ou, então quando há sucesso terapêutico inquestionável e a terapia convencional é pouco eficaz.

* * Desfechos substitutos são considerados validados quando há demonstração, em ECR de longa duração, de que mudanças entre os desfechos substitutos e clínicos são correlacionadas e de que o substituo capta plenamente todos os efeitos da terapia (ex.: contagem de CD4 foi validada como preditiva de incidência de mortalidade na AIDS).

Por conseguinte, essas intervenções ainda não foram estudadas de forma sistematizada, sendo então classificadas como intervenções cuja eficácia é desconhecida (evidência $\bullet V)^{20}$.

\section{Intervenções comportamentais}

As intervenções cognitivo-comportamentais têm sido frequentemente mencionadas como parte do tratamento da SCN. No entanto, ainda faltam estudos comparativos com metodologia apropriada para avaliar o nível de eficácia das intervenções, para definir nível de evidência e grau de recomendação. O nível de evidência atual é $\bullet$.

\section{Tratamento farmacológico}

Para analisar as evidências farmacológicas relacionadas ao tratamento da SCN foram consultadas as seguintes bases de dados: Medline (site Pubmed), Lilacs, National Guideline Clearinghouse e DARE.

No Medline (site Pubmed), utilizando as palavras-chave night eating syndrome, definindo como desfecho de interesse e como fator qualquer intervenção farmacológica. Foram encontrados 64 artigos nos últimos 10 anos. Usando os limites para publicações dos últimos 10 anos em língua inglesa, espanhola e portuguesa, com limites para ensaio clínico randomizado realizados em humanos, foram encontrados 7 artigos. Quando o limite para o tipo de estudo foi revisão, localizaram-se 6 artigos. Utilizando as palavras night eating syndrome and SSRI (selective serotonin reuptake inhibitors), sem limitações, foram encontrados 3 artigos. Utilizando as palavras night eating syndrome and anorectics foram encontrados 2 artigos. Utilizando as palavras night eating syndrome and melatonin foram encontrados 6 artigos. Utilizando as palavras night eating syndrome and tryptophan foi encontrado 1 artigo. Utilizando as palavras nighteating syndromeand benzodiazepines foi encontrado 1 artigo. Utilizando as palavras night eating syndrome and antidepressants tryciclics não foram localizados artigos.

No Lilacs, utilizando as palavras night eating syndrome, limitado para publicações dos últimos 10 anos, foi encontrado 1 artigo. No National Guideline Clearinghouse e no DARE 
utilizando as palavras night eating syndrome não foram encontrados artigos. Ao término dessa busca, foram selecionados e incluídos ensaios clínicos que apresentam como desfecho síndrome do comer noturno e como fatores intervenções farmacológicas. Restaram apenas 4 artigos, que estão sumarizados na Quadro 4.

\section{I S C U S S Ã O}

O reconhecimento e o tratamento da Síndrome do Comer Noturno têm crescido sendo um importante caminho para investigar a relação entre esta síndrome e o processo saúde-doença. De acordo com evidências recentes, a SCN parece apresentar forte associação à obesidade, ao diabetes tipo II e à pobre qualidade de vida $25-27,17$. Sobretudo, apontam-na como um fator que contribui fortemente com o desenvolvimento do sobrepeso e da obesidade. Por este motivo, este transtorno da vida moderna suscita o interesse nos meandros da saúde pública. Mas, ainda existe longo percurso a ser percorrido para compreender sua gênese e as relações intrínsecas desta com outros processos fisiopatogênicos. Tendo em conta que esta síndrome está vinculada ao controle da fome e da saciedade e à dessincronização entre o ritmo alimentar e o ritmo sono/vigília, a compreensão do seu processo gênico poderá demonstrar o impacto da dessincronização dos ritmos circadianos da alimentação no processo saúde-doença. Também, poderá auxiliar a compreensão de fatores implicados no índice crescente de obesidade da sociedade moderna.

Certamente, dentre os passos mais importantes para a universalização da linguagem do que compreende a SCN será o estabelecimento de consenso sobre os critérios diagnósticos que, embora revisados e modificados, não foram ainda formalmente validados. Além disso, futuros estudos deverão explorar critérios preditivos para a SCN. Outro passo importante será a incorporação

Quadro 4. Estudos com intervenções farmacológicas para tratamento de transtornos alimentares e nível de evidência.

\begin{tabular}{|c|c|c|c|c|c|c|}
\hline Referência & $\begin{array}{l}\text { Tipo de estudo e } \\
\text { níveis de evidência } \\
\text { Estudo (n) }\end{array}$ & Tratamento & Diagnóstico & Conclusão & Comentários & Tipo de amostra \\
\hline Winkelman ${ }^{28}$ & $\begin{array}{c}\text { Série de casos } \\
\quad(\downarrow \text { IV })\end{array}$ & $\begin{array}{c}\text { Topiramato } \\
\text { por } 8 \text { semanas }\end{array}$ & $\begin{array}{l}\text { SCN }(n=2) \\
\text { SRED }(n=2)\end{array}$ & $\begin{array}{l}50 \% \text { de sucesso } \\
(\downarrow \text { média } 11,1 \mathrm{Kg} \text { ) }\end{array}$ & $\begin{array}{l}\text { Medicações } \\
\text { psiquiátricas e } \\
\text { p/transtornos } \\
\text { do sono }\end{array}$ & SCN ou SRED \\
\hline $\begin{array}{l}\text { O'Reardon } \\
\text { et al. }{ }^{12}\end{array}$ & $\begin{array}{c}\text { Série de casos, } \\
(\bullet \mathrm{VI})\end{array}$ & $\begin{array}{l}\text { Sertralina por } \\
12 \text { semanas }\end{array}$ & $\begin{array}{c}\text { Obesos e obeso } \\
\text { mórbidos }\end{array}$ & $\begin{array}{c}\text { Em } 5 \text { sujeitos } \\
\text { controle dos } \\
\text { sintomas e peso }\end{array}$ & & $\begin{array}{l}\text { SCN Depressão, } \\
\text { Sem apneia do } \\
\text { sono; não } \\
\text { trabalhar à noite }\end{array}$ \\
\hline Spaggiari et al. ${ }^{29}$ & $\begin{array}{c}9 \text { Série de casos, } \\
(\bullet \mathrm{VI})\end{array}$ & $\begin{array}{c}\text { Fenfluramina } \\
\text { durante 6-15 } \\
\text { meses }\end{array}$ & $\begin{array}{l}\text { Sujeitostios com } \\
\text { ingestão compulsi- } \\
\text { va de alimentos }\end{array}$ & $\begin{array}{c}\text { 85,7\%6 redução } \\
\text { no } n^{\circ} \text { episódios } \\
\text { do comer noturno } \\
\text { e } \downarrow \text { na quantida- } \\
\text { de energética in- } \\
\text { gerida }\end{array}$ & & $\begin{array}{c}\text { Sujeitos deveriam } \\
\text { apresentar } \\
\text { acordar noturno } \\
\text { com comer } \\
\text { compulsivo }\end{array}$ \\
\hline $\begin{array}{l}\text { O'Reardon } \\
\text { et al. }{ }^{30}\end{array}$ & $\begin{array}{c}\text { ECRDCECRDC } \\
(\bullet \mathrm{II})\end{array}$ & Sertralina & $\begin{array}{c}\text { SCN Sintomas } \\
\text { depressivos leves }\end{array}$ & $\begin{array}{c}\text { Grupos } \\
\text { sertralina (71\%) } \\
\text { vs. placebo (18\%) } \\
\downarrow \text { peso, sintomas } \\
\text { de SCN e } \\
\text { qualidade de vida }\end{array}$ & $\begin{array}{c}\text { Estudo com boa } \\
\text { qualidade } \\
\text { metodológica } \\
\text { Resultados } \\
\text { passíveis de } \\
\text { aplicação clínica }\end{array}$ & $\begin{array}{l}\text { Excluídos com } \\
\text { diagnostico } \\
\text { psiquiátrico }\end{array}$ \\
\hline
\end{tabular}

SCN: síndrome do comer noturno; SRED: sleep-related eating disorder; ECRDC: ensaio clinico randomizado duplo-cego. 
dos critérios diagnósticos nos manuais de classificação diagnóstica, como o DSM e a CID-10. No que tange ao tratamento, serão necessários ensaios clínicos randomizados, duplo-cegos, controlados para que se possa estabelecer uma terapêutica baseada em adequado nível de evidência, e que permita mensurar seu real impacto terapêutico para o indivíduo e para a sociedade.

\section{OLABORADORES}

A.B.C. HARB realizou a busca, a leitura, a análise crítica para a revisão sistemática e posterior comparação e análise de artigos a serem incluídos na redação do artigo. W. CAUMO realizou a revisão e a análise do tratamento farmacológico. P. RAUPP realizou a busca, a leitura, a análise crítica para a revisão sistemática e posterior comparação e análise de artigos a serem incluídos na redação do artigo. M.P.L. HIDALGO realizou a busca, a leitura, a análise crítica para a revisão sistemática e posterior comparação e análise de artigos a serem incluídos na redação do artigo.

\section{REFER Ê N CIAS}

1. Kelly C, Allison AJSM, Sara L. Overcoming Night Eating Syndrome. In: Publications NH, editor. Overcoming night eating syndrome. A Step-by-step guide to breaking the cycle. Oakland (CA): New Harbinger Publications; 2004. v.1, p.192.

2. Hidalgo MP, Camozzato A, Cardoso L, Preussler C, Nunes CE, Tavares R, et al. Evaluation of behavioral states among morning and evening active healthy individuals. Br J Med Biol Res. 2002; 35:837-42.

3. Waterhouse J, Buckley P, Edwards B, Reilly T. Measurement of, and some reasons for, differences in eating habits between night and day workers. Chronobiol Int. 2003; 20(6):1075-92.

4. Harthoorn LF, Sane A, Nethe M, van Heerikhuize JJ. Multi-transcriptional profiling of melaninconcentrating hormone and orexin-containing neurons. Cell Mol Neurobiol. 2005; 25(8): 1209-23.

5. Shea SA, Hilton MF, Orlova C, Ayers RT, Mantzoros CS. Independent circadian and sleep/wake regulation of adipokines and glucose in humans. J Clin Endocrinol Metab. 2005; 90(5):2537-44.

6. Birketvedt GS, Sundsfjord J, Florholmen JR. Hypothalamic-pituitary-adrenal axis in the night eating syndrome. Am J Physiol Endocrinol Metab. 2002; 282(2):E366-9.

7. Birketvedt GS, Florholmen J, Sundsfjord J, Osterud B, Dinges D, Bilker W, Stunkard A. Behavioral and neuroendocrine characteristics of the night-eating syndrome. JAMA. 1999; 282(7):657-63.

8. Stunkard AJ, Allison KC. Two forms of disordered eating in obesity: binge eating and night eating. Int J Obes Relat Metab Disord. 2003; 27(1):1-12.

9. Stunkard AJ, Grace WJ, Wolff HG. The night-eating syndrome: a pattern of food intake among certain obese patients. Am J Med. 1955; 19(1):78-86.

10. Qin LQ, Li J, Wang Y, Wang J, Xu JY, Kaneko T. The effects of nocturnal life on endocrine circadian patterns in healthy adults. Life Sci. 2003; 73(19): 2467-75.

11. Stunkard A, Berkowitz R, Wadden T, Tanrikut C, Reiss $E$, Young L. Binge eating disorder and the night-eating syndrome. Int J Obes Relat Metab Disord. 1996; 20(1):1-6.

12. O'Reardon JP, Stunkard AJ, Allison KC. Clinical trial of sertraline in the treatment of night eating syndrome. Int J Eat Disord. 2004; 35(1):16-26.

13. Stunkard AJ, Allison KC, O'Reardon JP. The night eating syndrome: a progress report. Appetite. 2005; 45(2):182-6.

14. Gluck ME, Geliebter A, Satov T. Night eating syndrome is associated with depression, low selfesteem, reduced daytime hunger, and less weight loss in obese outpatients. Obes Res. 2001; 9(4): 264-7.

15. Marshall HM, Allison KC, O’Reardon JP, Birketvedt $G$, Stunkard AJ. Night eating syndrome among nonobese persons. Int J Eat Disord. 2004; 35(2): 217-22.

16. Striegel-Moore RH, Thompson D, Franko DL, Barton B, Affenito S, Schreiber GB, Daniels SR. Definitions of night eating in adolescent girls. Obes Res. 2004; 12(8):1311-21.

17. Allison KC, Crow SJ, Reeves RR, West DS, Foreyt JP, Dilillo VG, et al. Binge eating disorder and night eating syndrome in adults with type 2 diabetes. Obesity. 2007; 15(5):1287-93.

18. Allison KC, Wadden TA, Sarwer DB, Fabricatore $A N$, Crerand CE, Gibbons LM, et al. Night eating syndrome and binge eating disorder among persons seeking bariatric surgery: prevalence and related features. Surg Obes Relat Dis. 2006; 2(2):153-8.

19. Aronoff NJ, Geliebter A, Zammit G. Gender and body mass index as related to the night-eating syndrome in obese outpatients. J Am Diet Assoc. 2001; 101(1):102-4. 
20. O'Reardon JP, Peshek A, Allison KC. Night eating syndrome: diagnosis, epidemiology and management. CNS Drugs. 2005; 19(12):997-1008.

21. Lundgren JD, Allison KC, Crow S, O'Reardon JP, Berg KC, Galbraith J, et al. Prevalence of the night eating syndrome in a psychiatric population. Am J Psychiatry. 2006; 163(1):156-8.

22. Martina Z, Melissa AB, Carlos HS, James EM. Night time eating: a review of the literature. Eur Eating Disord Rev. 2003; 11(1):7-24.

23. Allison KC, Grilo CM, Masheb RM, Stunkard AJ. Binge eating disorder and night eating syndrome: a comparative study of disordered eating. J Consult Clin Psychol. 2005; 73(6):1107-15.

24. Yager J. Nocturnal eating syndromes: to sleep, perchance to eat. JAMA. 1999; 282(7):689-90.

25. Turner RW. Coronary heart disease: the size and nature of the problem. Postgrad Med J. 1980; 56(658):538-47.

26. Zimmet PZ, King HO, Bjorntorp SP. Obesity, hypertension, carbohydrate disorders and the risk of chronic diseases. Is there any epidemiological evidence for integrated prevention programmes? Med J Aust. 1986; 145(6):256-9, 62.

27. Martins IS, Coelho LT, Mazzilli RN, Singer JM, Souza $C U$, Antonieto Junior $A E$, et al. Atherosclerotic cardiovascular diseases, dyslipidemia, hypertension, obesity, and diabetes mellitus in a population of the metropolitan area of southeastern Brazil. Rev Saúde Pública. 1993; 27(4): 250-61.

28. Winkelman JW. Treatment of nocturnal eating syndrome and sleep-related eating disorder with topiramate. Sleep Med. 2003; 4(3):243-6.

29. Spaggiari MC, Granella F, Parrino L, Marchesi C, Melli I, Terzano MG. Nocturnal eating syndrome in adults. Sleep. 1994; 17(4):339-44.

30. O'Reardon JP, Allison KC, Martino NS, Lundgren JD, Heo M, Stunkard AJ. A randomized, placebocontrolled trial of sertraline in the treatment of night eating syndrome. Am J Psychiatry. 2006; 163(5):893-8.

Recebido em: 28/1/2008

Versão final reapresentada em: 15/11/2008 Aprovado em: 6/10/2009 